\title{
Positive solutions of the three-point boundary value problem for fractional-order differential equations with an advanced argument
}

\author{
Guotao Wang $^{1 *}$, SK Ntouyas ${ }^{2}$ and Lihong Zhang ${ }^{1}$
}

\footnotetext{
* Correspondence: wgt2512@163. com

${ }^{1}$ School of Mathematics and Computer Science, Shanxi Normal University, Linfen, Shanxi 041004, P. R. China

Full list of author information is available at the end of the article
}

\author{
Abstract \\ In this article, we consider the existence of at least one positive solution to the \\ three-point boundary value problem for nonlinear fractional-order differential \\ equation with an advanced argument

$$
\left\{\begin{array}{l}
{ }^{C} D^{\alpha} u(t)+a(t) f(u(\theta(t)))=0, \quad 0<t<1, \\
u(0)=u^{\prime \prime}(0)=0, \quad \beta u(\eta)=u(1)
\end{array}\right.
$$ \\ where $2<\alpha \leq 3,0<\eta<1,0<\beta<\frac{1}{{ }^{\prime}}{ }^{c} D^{\alpha}$ is the Caputo fractional derivative. Using \\ the well-known Guo-Krasnoselskii fixed point theorem, sufficient conditions for the \\ existence of at least one positive solution are established. \\ MSC (2010): 34A08; 34B18; 34K37.
}

Keywords: Positive solution, Three-point boundary value problem, Fractional differential equations, Guo-Krasnoselskii fixed point theorem, Cone

\section{Introduction}

The study of three-point BVPs for nonlinear integer-order ordinary differential equations was initiated by Gupta [1]. Many authors since then considered the existence and multiplicity of solutions (or positive solutions) of three-point BVPs for nonlinear integer-order ordinary differential equations. To identify a few, we refer the reader to [2-13] and the references therein.

Fractional differential equations arise in many engineering and scientific disciplines as the mathematical modeling of systems and processes in the fields of physics, chemistry, aerodynamics, electrodynamics of complex medium, polymer rheology, etc. [14-17]. In fact, fractional-order models have proved to be more accurate than integerorder models, i.e., there are more degrees of freedom in the fractional-order models. In consequence, the subject of fractional differential equations is gaining much importance and attention. For details, see [18-36] and the references therein.

Differential equations with deviated arguments are found to be important mathematical tools for the better understanding of several real world problems in physics, mechanics, engineering, economics, etc. $[37,38]$. In fact, the theory of integer order differential equations with deviated arguments has found its extensive applications in realistic mathematical modelling of a wide variety of practical situations and has emerged

(c) 2011 Wang et al; licensee Springer. This is an Open Access article distributed under the terms of the Creative Commons Attribution License (http://creativecommons.org/licenses/by/2.0), which permits unrestricted use, distribution, and reproduction in any medium provided the original work is properly cited. 
as an important area of investigation. For the general theory and applications of integer order differential equations with deviated arguments, we refer the reader to the references [39-45].

As far as we know, fractional order differential equations with deviated arguments have not been much studied and many aspects of these equations are yet to be explored. For some recent work on equations of fractional order with deviated arguments, see [46-48] and the references therein. In this article, we consider the following three-point BVPs for nonlinear fractional-order differential equation with an advanced argument

$$
\left\{\begin{array}{l}
{ }^{C} D^{\alpha} u(t)+a(t) f(u(\theta(t)))=0, \quad 0<t<1, \\
u(0)=u^{\prime \prime}(0)=0, \quad \beta u(\eta)=u(1),
\end{array}\right.
$$

where $2<\alpha \leq 3,0<\eta<1,0<\beta<\frac{1}{\eta},{ }^{C} D^{\alpha}$ is the Caputo fractional derivative and $f$ : $[0, \infty) \rightarrow[0, \infty)$ is a continuous function.

By a positive solution of (1.1), one means a function $u(t)$ that is positive on $0<t<1$ and satisfies (1.1).

Our purpose here is to give the existence of at least one positive solution to problem (1.1), assuming that

$\left(H_{1}\right): a \in C([0,1],[0, \infty))$ and $a$ does not vanish identically on any subinterval.

$\left(H_{2}\right)$ : The advanced argument $\theta \in C((0,1),(0,1))$ and $t \leq \theta(t) \leq 1, \forall t \in(0,1)$.

Let $E=C[0,1]$ be the Banach space endowed with the sup-norm. Set

$$
f_{0}=\lim _{u \rightarrow 0+} \frac{f(u)}{u}, \quad f_{\infty}=\lim _{u \rightarrow \infty} \frac{f(u)}{u} .
$$

The main results of this paper are as follows.

Theorem 1.1 Assume that $\left(H_{1}\right)$ and $\left(H_{2}\right)$ hold. If $f_{0}=\infty$ and $f_{\infty}=0$, then problem (1.1) has at least one positive solution.

Theorem 1.2 Assume that $\left(H_{1}\right)$ and $\left(H_{2}\right)$ hold. If $f_{0}=\infty$ and $f_{\infty}=\infty$, then problem (1.1) has at least one positive solution.

Remark 1.1 It is worth mentioning that the conditions of our theorems are easily to verify, so they are applicable to a variety of problems, see Examples 4.1 and 4.2.

The proof of our main results is based upon the following well-known Guo-Krasnoselskii fixed point theorem:

Theorem 1.3 [49] Let $E$ be a Banach space, and let $P \subset E$ be a cone. Assume that $\Omega_{1}, \Omega_{2}$ are open subsets of $E$ with $0 \in \Omega_{1}, \bar{\Omega}_{1} \subset \Omega_{2}$, and let $T: P \cap\left(\bar{\Omega}_{2} \backslash \Omega_{1}\right) \rightarrow$ Pbe a completely continuous operator such that

(i) $\|T u\| \geq\|u\|, u \in P \cap \partial \Omega_{1}$, and $\|T u\| \leq\|u\|, u \in P \cap \partial \Omega_{2}$; or

(ii) $\|T u\| \leq\|u\|, u \in P \cap \partial \Omega_{1}$, and $\|T u\| \geq\|u\|, u \in P \cap \partial \Omega_{2}$.

Then $T$ has a fixed point in $P \cap\left(\bar{\Omega}_{2} \backslash \Omega_{1}\right)$.

\section{Preliminaries}

For the reader's convenience, we present some necessary definitions from fractional calculus theory and Lemmas.

Definition 2.1 For a function $f:[0, \infty) \rightarrow \mathbb{R}$, the Caputo derivative of fractional order $\alpha$ is defined as 


$$
{ }^{C} D^{\alpha} f(t)=\frac{1}{\Gamma(n-\alpha)} \int_{0}^{t}(t-s)^{n-\alpha-1} f^{(n)}(s) \mathrm{d} s, \quad n-1<\alpha<n, n=[\alpha]+1,
$$

where $[\alpha]$ denotes the integer part of real number $\alpha$.

Definition 2.2 The Riemann-Liouville fractional integral of order $\alpha$ is defined as

$$
I^{\alpha} f(t)=\frac{1}{\Gamma(\alpha)} \int_{0}^{t}(t-s)^{\alpha-1} f(s) \mathrm{d} s, \quad \alpha>0,
$$

provided the integral exists.

Definition 2.3 The Riemann-Liouville fractional derivative of order $\alpha$ for a function $f$ $(t)$ is defined by

$$
D^{\alpha} f(t)=\frac{1}{\Gamma(n-\alpha)}\left(\frac{d}{d t}\right)^{n} \int_{0}^{t}(t-s)^{n-\alpha-1} f(s) \mathrm{d} s, \quad n=[\alpha]+1,
$$

provided the right-hand side is pointwise defined on $(0, \infty)$.

Lemma 2.1 [15] Let $\alpha>0$, then fractional differential equation

$$
{ }^{C} D^{\alpha} u(t)=0
$$

has solution

$$
u(t)=C_{0}+C_{1} t+C_{2} t^{2}+\cdots+C_{n-1} t^{n-1}, C_{i} \in \mathbb{R}, \quad i=0,1,2, \ldots, n-1,
$$

where $n$ is the smallest integer greater than or equal to $\alpha$.

Lemma 2.2 [15] Let $\alpha>0$, then

$$
I^{\alpha C} D^{\alpha} u(t)=u(t)+C_{0}+C_{1} t+C_{2} t^{2}+\cdots+C_{n-1} t^{n-1}
$$

for some $C_{i} \in \mathbb{R}, i=0,1,2, \ldots, N-1$, where $N$ is the smallest integer greater than or equal to $\alpha$.

Lemma 2.3 Let $2<\alpha \leq 3,1 \neq \beta \eta$. Assume $y(t) \in C[0,1]$, then the following problem

$$
\begin{aligned}
& { }^{C} D^{\alpha} u(t)+\gamma(t)=0, \quad 0<t<1, \\
& u(0)=u^{\prime \prime}(0)=0, \quad \beta u(\eta)=u(1),
\end{aligned}
$$

has a unique solution

$$
u(t)=-\int_{0}^{t} \frac{(t-s)^{\alpha-1}}{\Gamma(\alpha)} \gamma(s) \mathrm{d} s+\frac{1}{1-\beta \eta} \int_{0}^{1} \frac{t(1-s)^{\alpha-1}}{\Gamma(\alpha)} \gamma(s) \mathrm{d} s-\frac{\beta}{1-\beta \eta} \int_{0}^{\eta} \frac{t(\eta-s)^{\alpha-1}}{\Gamma(\alpha)} \gamma(s) \mathrm{d} s .
$$

Proof. We may apply Lemma 2.2 to reduce Equation (2.1) to an equivalent integral equation

$$
u(t)=-I^{\alpha} \gamma(t)-b_{1}-b_{2} t-b_{3} t^{2}=-\int_{0}^{t} \frac{(t-s)^{\alpha-1}}{\Gamma(\alpha)} y(s) \mathrm{d} s-b_{1}-b_{2} t-b_{3} t^{2},
$$

for some $b_{1}, b_{2}, b_{3} \in \mathbb{R}$. 
In view of the relation ${ }^{C} D^{\alpha} I^{\alpha} u(t)=u(t)$ and $I^{\alpha} I^{\beta} u(t)=I^{\alpha+\beta} u(t)$ for $\alpha, \beta>0$, we can get that

$$
\begin{aligned}
& u^{\prime}(t)=-\int_{0}^{t} \frac{(t-s)^{\alpha-2}}{\Gamma(\alpha-1)} \gamma(s) \mathrm{d} s-b_{2}-2 b_{3} t . \\
& u^{\prime \prime}(t)=-\int_{0}^{t} \frac{(t-s)^{\alpha-3}}{\Gamma(\alpha-2)} \gamma(s) \mathrm{d} s-2 b_{3} .
\end{aligned}
$$

By $u(0)=u^{\prime \prime}(0)=0$, it follows $b_{1}=b_{3}=0$. Then by the condition $\beta u(\eta)=u(1)$, we have

$$
-b_{2}=\frac{1}{1-\beta \eta} \int_{0}^{1} \frac{(1-s)^{\alpha-1}}{\Gamma(\alpha)} \gamma(s) \mathrm{d} s-\frac{\beta}{1-\beta \eta} \int_{0}^{\eta} \frac{(\eta-s)^{\alpha-1}}{\Gamma(\alpha)} \gamma(s) \mathrm{d} s .
$$

Combine with (2.3), we get

$$
u(t)=-\int_{0}^{t} \frac{(t-s)^{\alpha-1}}{\Gamma(\alpha)} \gamma(s) \mathrm{d} s+\frac{1}{1-\beta \eta} \int_{0}^{1} \frac{t(1-s)^{\alpha-1}}{\Gamma(\alpha)} \gamma(s) \mathrm{d} s-\frac{\beta}{1-\beta \eta} \int_{0}^{\eta} \frac{t(\eta-s)^{\alpha-1}}{\Gamma(\alpha)} \gamma(s) \mathrm{d} s .
$$

This complete the proof.

Lemma 2.4 Let $2<\alpha \leq 3,0<\beta<\frac{1}{\eta}$. Assume $y \in C([0,1],[0, \infty))$, then the unique solution $u$ of (2.1) and (2.2) satisfies $u(t) \geq 0, \forall t \in[0,1]$.

Proof. By Lemma 2.3, we know that $u^{\prime \prime}(t)=-\int_{0}^{t} \frac{(t-s)^{\alpha-3}}{\Gamma(\alpha-2)} y(s) \mathrm{d} s \leq 0$. It means that the graph of $u(t)$ is concave down on $(0,1)$.

In addition,

$$
\begin{aligned}
u(1) & =-\int_{0}^{1} \frac{(1-s)^{\alpha-1}}{\Gamma(\alpha)} \gamma(s) \mathrm{d} s+\frac{1}{1-\beta \eta} \int_{0}^{1} \frac{(1-s)^{\alpha-1}}{\Gamma(\alpha)} \gamma(s) \mathrm{d} s-\frac{\beta}{1-\beta \eta} \int_{0}^{\eta} \frac{(\eta-s)^{\alpha-1}}{\Gamma(\alpha)} \gamma(s) \mathrm{d} s \\
& =\frac{\beta \eta}{1-\beta \eta} \int_{0}^{1} \frac{(1-s)^{\alpha-1}}{\Gamma(\alpha)} \gamma(s) \mathrm{d} s-\frac{\beta}{1-\beta \eta} \int_{0}^{\eta} \frac{(\eta-s)^{\alpha-1}}{\Gamma(\alpha)} \gamma(s) \mathrm{d} s \\
& \geq \frac{\beta}{1-\beta \eta}\left[\int_{0}^{\eta} \frac{\eta(1-s)^{\alpha-1}}{\Gamma(\alpha)} \gamma(s) \mathrm{d} s-\int_{0}^{\eta} \frac{(\eta-s)^{\alpha-1}}{\Gamma(\alpha)} \gamma(s) \mathrm{d} s\right] \\
& =\frac{\beta}{(1-\beta \eta) \Gamma(\alpha)} \int_{0}^{\eta}\left[\eta(1-s)^{\alpha-1}-(\eta-s)^{\alpha-1}\right] y(s) \mathrm{d} s \\
& \geq \frac{\beta}{(1-\beta \eta) \Gamma(\alpha)} \int_{0}^{\eta}\left[(\eta-\eta s)^{\alpha-1}-(\eta-s)^{\alpha-1}\right] y(s) \mathrm{d} s \geq 0
\end{aligned}
$$

Combine with $u(0)=0$, it follows $u(t) \geq 0, \forall t \in[0,1]$.

Lemma 2.5 Let $2<\alpha \leq 3,0<\beta<\frac{1}{\eta}$. Assume $y \in C([0,1],[0, \infty))$, then the unique solution $u$ of (2.1) and (2.2) satisfies 


$$
\inf _{t \in[\eta, 1]} u(t) \geq \gamma\|u\|
$$

where $\gamma=\min \left\{\beta \eta, \frac{\beta(1-\eta)}{1-\beta \eta}, \eta\right\}$.

Proof. Note that $u^{\prime \prime}(t) \leq 0$, by applying the concavity of $u$, the proof is easy. So we omit it.

\section{Proofs of main theorems}

Define the operator $T: C[0,1] \rightarrow C[0,1]$ as follows,

$$
\begin{aligned}
T u(t)= & -\int_{0}^{t} \frac{(t-s)^{\alpha-1}}{\Gamma(\alpha)} a(s) f(u(\theta(s))) \mathrm{d} s+\frac{1}{1-\beta \eta} \int_{0}^{1} \frac{t(1-s)^{\alpha-1}}{\Gamma(\alpha)} a(s) f(u(\theta(s))) \mathrm{d} s \\
& -\frac{\beta}{1-\beta \eta} \int_{0}^{\eta} \frac{t(\eta-s)^{\alpha-1}}{\Gamma(\alpha)} a(s) f(u(\theta(s))) d s .
\end{aligned}
$$

Then the problem (1.1) has a solution if and only if the operator $T$ has a fixed point. Define the cone $P=\left\{u \mid u \in C[0,1], u \geq 0, \inf _{t \in[\eta, 1]} u(\theta(t)) \geq \gamma\|u\|\right\}$, where $\gamma=\min \left\{\beta \eta, \frac{\beta(1-\eta)}{1-\beta \eta}, \eta\right\}$.

Proof of Theorem 1.1. The operator $T$ is completely continuous. Obviously, $T$ is continuous.

Let $\Omega \subset C[0,1]$ be bounded, then there exists a constant $K>0$ such that $\| a(t) f(u$ $(\theta(t)) \| \leq K, \forall u \in \Omega$. Thus, we have

$$
\begin{aligned}
\operatorname{Tu}(t) & \leq \frac{1}{1-\beta \eta} \int_{0}^{1} \frac{(1-s)^{\alpha-1}}{\Gamma(\alpha)} a(s) f(u(\theta(s))) \mathrm{d} s \\
& \leq \frac{K}{1-\beta \eta} \int_{0}^{1} \frac{(1-s)^{\alpha-1}}{\Gamma(\alpha)} \mathrm{d} s \\
& =\frac{K}{(1-\beta \eta) \Gamma(\alpha+1)},
\end{aligned}
$$

which implies $\|T u\| \leq \frac{K}{(1-\beta \eta) \Gamma(\alpha+1)}$.

On the other hand, we have

$$
\begin{aligned}
\left|(T u)^{\prime}(t)\right| \leq & \int_{0}^{t} \frac{(t-s)^{\alpha-2}}{\Gamma(\alpha-1)} a(s) f(u(\theta(s))) \mathrm{d} s+\frac{1}{1-\beta \eta} \int_{0}^{1} \frac{(1-s)^{\alpha-1}}{\Gamma(\alpha)} a(s) f(u(\theta(s))) \mathrm{d} s \\
& +\frac{\beta}{1-\beta \eta} \int_{0}^{\eta} \frac{(\eta-s)^{\alpha-1}}{\Gamma(\alpha)} a(s) f(u(\theta(s))) \mathrm{d} s \\
\leq & K \int_{0}^{1} \frac{(1-s)^{\alpha-2}}{\Gamma(\alpha-1)} \mathrm{d} s+\frac{K}{1-\beta \eta} \int_{0}^{1} \frac{(1-s)^{\alpha-1}}{\Gamma(\alpha)} \mathrm{d} s+\frac{K \beta}{1-\beta \eta} \int_{0}^{1} \frac{(1-s)^{\alpha-1}}{\Gamma(\alpha)} \mathrm{d} s \\
= & \frac{K}{\Gamma(\alpha)}+\frac{(1+\beta) K}{(1-\beta \eta) \Gamma(\alpha+1)}:=M .
\end{aligned}
$$


Hence, for each $u \in \Omega$, let $t_{1}, t_{2} \in[0,1], t_{1}<t_{2}$, we have

$$
\left|(T u)\left(t_{2}\right)-(T u)\left(t_{1}\right)\right| \leq \int_{t_{1}}^{t_{2}}\left|(T u)^{\prime}(s)\right| \mathrm{d} s \leq M\left(t_{2}-t_{1}\right) .
$$

So, $T$ is equicontinuous. The Arzela-Ascoli Theorem implies that $T: C[0,1] \rightarrow C[0$, $1]$ is completely continuous.

Since $t \leq \theta(t) \leq 1, t \in(0,1)$, then

$$
\inf _{t \in[\eta, 1]} u(\theta(t)) \geq \inf _{t \in[\eta, 1]} u(t) \geq \gamma\|u\| .
$$

Thus, Lemmas 2.5 and 3.2 show that $T P \subset P$. Then, $T: P \rightarrow P$ is completely continuous.

In view of $f_{0}=\infty$, there exists a constant $\rho_{1}>0$ such that $f(u) \geq \delta_{1} u$ for $0<u<\rho_{1}$, where $\delta_{1}>0$ satisfies

$$
\frac{\eta \delta_{1} \gamma}{(1-\beta \eta)} \int_{\eta}^{1} \frac{(1-s)^{\alpha-1}}{\Gamma(\alpha)} a(s) \mathrm{d} s \geq 1 .
$$

Take $u \in P$, such that $\|u\|=\rho_{1}$. Then, we have

$$
\begin{aligned}
&\|T u\| \geq T u(\eta) \\
&=-\int_{0}^{\eta} \frac{(\eta-s)^{\alpha-1}}{\Gamma(\alpha)} a(s) f(u(\theta(s))) \mathrm{d} s+\frac{1}{1-\beta \eta} \int_{0}^{1} \frac{\eta(1-s)^{\alpha-1}}{\Gamma(\alpha)} a(s) f(u(\theta(s))) \mathrm{d} s \\
&-\frac{\beta}{1-\beta \eta} \int_{0}^{\eta} \frac{\eta(\eta-s)^{\alpha-1}}{\Gamma(\alpha)} a(s) f(u(\theta(s))) \mathrm{d} s \\
&=-\frac{1}{1-\beta \eta} \int_{0}^{\eta} \frac{(\eta-s)^{\alpha-1}}{\Gamma(\alpha)} a(s) f(u(\theta(s))) \mathrm{d} s+\frac{\eta}{1-\beta \eta} \int_{0}^{1} \frac{(1-s)^{\alpha-1}}{\Gamma(\alpha)} a(s) f(u(\theta(s))) \mathrm{d} s \\
&=-\frac{1}{1-\beta \eta} \int_{0}^{\eta} \frac{(\eta-s)^{\alpha-1}}{\Gamma(\alpha)} a(s) f(u(\theta(s))) \mathrm{d} s+\frac{\eta}{1-\beta \eta} \int_{0}^{\eta} \frac{(1-s)^{\alpha-1}}{\Gamma(\alpha)} a(s) f(u(\theta(s))) \mathrm{d} s \\
&+\frac{\eta}{1-\beta \eta} \int_{\eta}^{1} \frac{(1-s)^{\alpha-1}}{\Gamma(\alpha)} a(s) f(u(\theta(s))) \mathrm{d} s \\
& \geq-\frac{1}{1-\beta \eta} \int_{0}^{\eta} \frac{(\eta-s)^{\alpha-1}}{\Gamma(\alpha)} a(s) f(u(\theta(s))) \mathrm{d} s+\frac{1}{1-\beta \eta} \int_{0}^{\eta} \frac{(\eta-\eta)^{\alpha-1}}{\Gamma(\alpha)} a(s) f(u(\theta(s))) \mathrm{d} s \\
&+\frac{\eta}{1-\beta \eta} \int_{\eta}^{1} \frac{(1-s)^{\alpha-1}}{\Gamma(\alpha)} a(s) f(u(\theta(s))) \mathrm{d} s \\
& \geq \frac{\eta}{1-\beta \eta} \int_{\eta}^{1} \frac{(1-s)^{\alpha-1}}{\Gamma(\alpha)} a(s) f(u(\theta(s))) \mathrm{d} s \\
& \geq \frac{\eta}{1-\beta \eta} \int_{\eta}^{1} \frac{(1-s)^{\alpha-1}}{\Gamma(\alpha)} a(s) \delta_{1} u(\theta(s)) \mathrm{d} s \\
& \geq \frac{\eta}{1-\beta \eta} \int_{\eta}^{1} \frac{(1-s)^{\alpha-1}}{\Gamma(\alpha)} a(s) \delta_{1} \gamma\|u\| \mathrm{d} s \\
&= \frac{\eta \delta_{1} \gamma}{(1-\beta \eta)} \int_{\eta}^{1} \frac{(1-s)^{\alpha-1}}{\Gamma(\alpha)} a(s) \mathrm{d} s\|u\| \geq\|u\| . \\
&
\end{aligned}
$$

Let $\Omega_{\rho 1}=\left\{u \in C 0[1] \mid\|u\|<\rho_{1}\right\}$. Thus, (3.4) shows $\|T u\| \geq\|u\|, u \in P \cap \partial \Omega_{\rho 1}$. 
Next, in view of $f_{\infty}=0$, there exists a constant $R>\rho_{1}$ such that $f(u) \leq \delta_{2} u$ for $u \geq R$, where $\delta_{2}>0$ satisfies

$$
\frac{\delta_{2}}{(1-\beta \eta)} \int_{0}^{1} \frac{(1-s)^{\alpha-1}}{\Gamma(\alpha)} a(s) \mathrm{d} s \leq 1 .
$$

We consider the following two cases.

Case one. $f$ is bounded, which implies that there exists a constant $r_{1}>0$ such that $f$ $(u) \leq r_{1}$ for $u \in[0, \infty)$. Now, we may choose $u \in P$ such that $\|u\|=\rho_{2}$, where $\rho_{2} \geq$ $\max \{\mu, R\}$.

Then we have

$$
\begin{aligned}
T u(t)= & -\int_{0}^{t} \frac{(t-s)^{\alpha-1}}{\Gamma(\alpha)} a(s) f(u(\theta(s))) \mathrm{d} s+\frac{1}{1-\beta \eta} \int_{0}^{1} \frac{t(1-s)^{\alpha-1}}{\Gamma(\alpha)} a(s) f(u(\theta(s))) \mathrm{d} s \\
& -\frac{\beta}{1-\beta \eta} \int_{0}^{\eta} \frac{t(\eta-s)^{\alpha-1}}{\Gamma(\alpha)} a(s) f(u(\theta(s))) \mathrm{d} s \\
\leq & \frac{1}{1-\beta \eta} \int_{0}^{1} \frac{(1-s)^{\alpha-1}}{\Gamma(\alpha)} a(s) f(u(\theta(s))) \mathrm{d} s \\
\leq & \frac{r_{1}}{(1-\beta \eta)} \int_{0}^{1} \frac{(1-s)^{\alpha-1}}{\Gamma(\alpha)} a(s) \mathrm{d} s \\
\triangleq & \mu \leq \rho_{2}=\|u\| .
\end{aligned}
$$

Case two. $f$ is unbounded, which implies then there exists a constant $\rho_{2}>\frac{R}{\gamma}>R$ such that $f(u) \leq f\left(\rho_{2}\right)$ for $0<u \leq \rho_{2}$ (note that $f \in C([0, \infty),[0, \infty)$ ). Let $u \in P$ such that $\|u\|=\rho_{2}$, we have

$$
\begin{aligned}
\operatorname{Tu}(t) & \leq \frac{1}{1-\beta \eta} \int_{0}^{1} \frac{(1-s)^{\alpha-1}}{\Gamma(\alpha)} a(s) f(u(\theta(s))) \mathrm{d} s \\
& \leq \frac{1}{1-\beta \eta} \int_{0}^{1} \frac{(1-s)^{\alpha-1}}{\Gamma(\alpha)} a(s) f\left(\rho_{2}\right) \mathrm{d} s \\
& \leq \frac{1}{1-\beta \eta} \int_{0}^{1} \frac{(1-s)^{\alpha-1}}{\Gamma(\alpha)} a(s) \delta_{2} \rho_{2} \mathrm{~d} s \\
& =\frac{\delta_{2}}{(1-\beta \eta)} \int_{0}^{1} \frac{(1-s)^{\alpha-1}}{\Gamma(\alpha)} a(s) \mathrm{d} s\|u\| \\
& \leq\|u\| .
\end{aligned}
$$

Hence, in either case, we may always let $\Omega_{\rho 2}=\left\{u \in C[0,1] \mid\|u\|<\rho_{2}\right\}$ such that $\|$ $T u\|\leq\| u \|$ for $u \in P \cap \partial \Omega_{\rho 2}$.

Thus, by the first part of Guo-Krasnoselskii fixed point theorem, we can conclude that (1.1) has at least one positive solution.

Proof of Theorem 1.2. Now, in view of $f_{0}=0$, there exists a constant $r_{1}>0$ such that $f$ $(u) \leq \tau_{1} u$ for $0<u<r_{1}$, where $\tau_{1}>0$ satisfies 


$$
\frac{\tau_{1}}{(1-\beta \eta)} \int_{0}^{1} \frac{(1-s)^{\alpha-1}}{\Gamma(\alpha)} a(s) \mathrm{d} s \leq 1 .
$$

Take $u \in P$, such that $\|u\|=r_{1}$. Then, we have

$$
\begin{aligned}
T u(t) & \leq \frac{1}{1-\beta \eta} \int_{0}^{1} \frac{(1-s)^{\alpha-1}}{\Gamma(\alpha)} a(s) f(u(\theta(s))) \mathrm{d} s \\
& \leq \frac{1}{1-\beta \eta} \int_{0}^{1} \frac{(1-s)^{\alpha-1}}{\Gamma(\alpha)} a(s) \tau_{1} u(\theta(s)) \mathrm{d} s \\
& \leq \frac{\tau_{1}}{(1-\beta \eta)} \int_{0}^{1} \frac{(1-s)^{\alpha-1}}{\Gamma(\alpha)} a(s) \mathrm{d} s\|u\| \\
& \leq\|u\| .
\end{aligned}
$$

Let $\Omega_{1}=\left\{u \in C[0,1]\|\| u \|<r_{1}\right\}$. Thus, (3.7) shows $\|T u\| \leq\|u\|, u \in P \cap \partial \Omega_{1}$.

Next, in view of $f_{\infty}=\infty$, there exists a constant $r_{2}>r_{1}$ such that $f(u) \geq \tau_{2} u$ for $u \geq r_{2}$, where $\tau_{2}>0$ satisfies

$$
\frac{\tau_{2} \eta \gamma}{(1-\beta \eta)} \int_{\eta}^{1} \frac{(1-s)^{\alpha-1}}{\Gamma(\alpha)} a(s) \mathrm{d} s \geq 1 .
$$

Let $\Omega_{2}=\left\{u \in C[0,1] \mid\|u\|<\rho_{2}\right\}$, where $\rho_{2}>\frac{r_{2}}{\gamma}>r_{2}$, then, $u \in P$ and $\|u\|=\rho_{2}$ implies

$$
\inf _{t \in[\eta, 1]} u(\theta(t)) \geq \gamma\|u\|>r_{2}
$$

and so

$$
\begin{aligned}
\|T u\| & \geq T u(\eta) \\
& \geq \frac{\eta}{1-\beta \eta} \int_{\eta}^{1} \frac{(1-s)^{\alpha-1}}{\Gamma(\alpha)} a(s) f(u(\theta(s))) \mathrm{d} s \\
& \geq \frac{\eta}{1-\beta \eta} \int_{\eta}^{1} \frac{(1-s)^{\alpha-1}}{\Gamma(\alpha)} a(s) \tau_{2} u(\theta(s)) \mathrm{d} s \\
& \geq \frac{\eta}{1-\beta \eta} \int_{\eta}^{1} \frac{(1-s)^{\alpha-1}}{\Gamma(\alpha)} a(s) \tau_{2} \gamma\|u\| \mathrm{d} s \\
& =\frac{\tau_{2} \eta \gamma}{(1-\beta \eta)} \int_{\eta}^{1} \frac{(1-s)^{\alpha-1}}{\Gamma(\alpha)} a(s) \mathrm{d} s\|u\| \geq\|u\| .
\end{aligned}
$$

This shows that $\|T u\| \geq\|u\|$ for $u \in P \cap \partial \Omega_{2}$.

Therefore, by the second part of Guo-Krasnoselskii fixed point theorem, we can conclude that (1.1) has at least one positive solution $u \in P \cap\left(\bar{\Omega}_{2} \backslash \Omega_{1}\right)$. 


\section{Examples}

Example 4.1 Consider the fractional differential equation

$$
\left\{\begin{array}{l}
{ }^{C} D^{\alpha} u(t)+e^{-t} f(u(\theta(t)))=0, \quad 0<t<1 \\
u(0)=u^{\prime \prime}(0)=0, \quad \beta u(\eta)=u(1)
\end{array}\right.
$$

where $2<\alpha \leq 3,0<\eta<1,0<\beta<\frac{1}{\eta}, \theta(t)=t^{\nu}, 0<v<1$ and

$$
f(u)= \begin{cases}\frac{\sin u}{u^{2}}, & 0 \leq u \leq \frac{\pi}{2}, \\ \frac{4 \sqrt{2 u}+\cos u}{\pi^{\frac{5}{2}}}, & u>\frac{\pi}{2} .\end{cases}
$$

Note that conditions $\left(H_{1}\right)$ and $\left(H_{2}\right)$ of Theorem 1.1 hold. Through a simple calculation we can get $f_{0}=\infty$ and $f_{\infty}=0$. Thus, by Theorem 1.1 , we can get that the problem (4.1) has at least one positive solution.

Example 4.2 Consider the fractional differential equation

$$
\begin{cases}{ }^{C} D^{\alpha} u(t)+a(t) f(u(\theta(t)))=0, & 0<t<1, \\ u(0)=u^{\prime \prime}(0)=0, & \beta u(\eta)=u(1),\end{cases}
$$

where $2<\alpha \leq 3,0<\eta<1,0<\beta<\frac{1}{\eta}, \theta(t)=\sqrt{t}, a(t)=e^{\tan t}$ and

$$
f(u)=u^{\frac{3}{2}} \ln (1+u)+u^{3+\sin u} .
$$

Obviously, it is not difficult to verify conditions $\left(H_{1}\right)$ and $\left(H_{2}\right)$ of Theorem 1.2 hold. Through a simple calculation we can get $f_{0}=0$ and $f_{\infty}=\infty$. Thus, by Theorem 1.2, we can get that the problem (4.2) has at least one positive solution.

Remark 4.1 In the above two examples, $\alpha, \beta, \eta$ could be any constants which satisfy $2<\alpha \leq 3,0<\eta<1,0<\beta<\frac{1}{\eta}$. For example, we can take $\alpha=2.5, \eta=0.5, \beta=1.5$.

\section{Author details}

'School of Mathematics and Computer Science, Shanxi Normal University, Linfen, Shanxi 041004, P. R. China ${ }^{2}$ Department of Mathematics, University of loannina, loannina 45110, Greece

\section{Authors' contributions}

GW completed the main part of this paper, SKN and LZ corrected the main theorems and gave two examples. All authors read and approved the final manuscript.

\section{Competing interests}

The authors declare that they have no competing interests.

Received: 12 December 2010 Accepted: 17 May 2011 Published: 17 May 2011

\section{References}

1. Gupta CP: Solvability of a three-point nonlinear boundary value problem for a second order ordinary differential equation. J Math Anal Appl 1992, 168:540-551.

2. Jankowski T: Positive solutions for three-point one-dimensional p-Laplacian boundary value problems with advanced arguments. Appl Math Comput 2009, 215:125-131.

3. Jankowski T: Solvability of three point boundary value problems for second order differential equations with deviating arguments. J Math Anal App/ 2005, 312:620-636.

4. Ma R: Multiplicity of positive solutions for second-order three-point boundary value problems. Comput Math Appl 2000, 40:193-204. 
5. Webb JRL: Positive solutions of some three point boundary value problems via fixed point index theory. Nonlinear Anal 2001, 47:4319-4332.

6. Raffoul YN: Positive solutions of three-point nonlinear second order boundary value problem. Electron I Qual Theory Differ Equ 2002, 15:1-11.

7. Xu X: Multiplicity results for positive solutions of some semi-positone three-point boundary value problems. J Math Anal Appl 2004, 291:673-689.

8. Ma R: Positive solutions of a nonlinear three-point boundary value problem. Electron J Differ Equ 1998, 34:1-8.

9. Marano SA: A remark on a second order three-point boundary value problem. J Math Anal Appl 1994, 183:518-522.

10. Feng W, Webb JRL: Solvability of three point boundary value problems at resonance. Nonlinear Anal 1997, 30(6):3227-3238.

11. Anderson D: Multiple positive solutions for a three-point boundary value problem. Math Comput Model 1998, 27(6):49-57.

12. Gupta CP, Trofimchuk SI: A sharper condition for the solvability of a three-point second order boundary value problem. J Math Anal Appl 1997, 205:586-597.

13. Pang $H$, Feng $M$, Ge W: Existence and monotone iteration of positive solutions for a three-point boundary value problem. Appl Math Lett 2008, 21:656-661.

14. Podlubny I: Fractional Differential Equations. Academic Press, San Diego; 1999.

15. Kilbas AA, Srivastava HM, Trujillo JJ: Theory and Applications of Fractional Differential Equations. In North-Holland Mathematics Studies. Volume 204. Elsevier, Amsterdam; 2006.

16. Sabatier J, Agrawal OP, Machado JAT, (eds.): Advances in Fractional Calculus: Theoretical Developments and Applications in Physics and Engineering. Springer, Dordrecht; 2007.

17. Lakshmikantham V, Leela S, Vasundhara Devi J: Theory of Fractional Dynamic Systems. Cambridge Academic Publishers, Cambridge; 2009.

18. Lakshmikantham V, Vatsala AS: Basic theory of fractional differential equations. Nonlin-ear Anal 2008, 69(8):2677-2682.

19. Lazarević MP, Spasić AM: Finite-time stability analysis of fractional order time-delay systems: Gronwall's approach. Math Comput Model 2009, 49:475-481.

20. Belmekki M, Nieto JJ, Rodriguez-Lopez R: Existence of periodic solution for a nonlinear fractional differential equation. Bound Value Probl 2009, 18, Article ID 324561.

21. Ahmad B, Nieto Jj: Existence results for a coupled system of nonlinear fractional differential equations with threepoint boundary conditions. Comput Math Appl 2009, 58:1838-1843.

22. Benchohra M, Hamani S, Ntouyas SK: Boundary value problems for differential equations with fractional order and nonlocal conditions. Nonlinear Anal 2009, 71:2391-2396.

23. Darwish MA, Ntouyas SK: Monotonic solutions of a perturbed quadratic fractional integral equation. Nonlinear Anal 2009, 71:5513-5521.

24. Zhang SQ: Positive solutions to singular boundary value problem for nonlinear fractional differential equation. Comput Math Appl 2010, 59:1300-1309.

25. Nieto JJ: Maximum principles for fractional differential equations derived from Mittag-Leffler functions. Appl Math Lett 2010, 23:1248-1251.

26. Bonilla B, Rivero M, Rodrguez-Germǎ L, Trujillo JJ: Fractional differential equations as alternative models to nonlinear differential equations. Appl Math Comput 2007, 187:79-88.

27. Balachandran K, Trujillo JJ: The nonlocal Cauchy problem for nonlinear fractional integrodifferential equations in Banach spaces. Nonlinear Anal 2010, 72:4587-4593.

28. Goodrich ChS: Existence of a positive solution to a class of fractional differential equations. Appl Math Lett 2010 23:1050-1055.

29. Bai ZB: On positive solutions of a nonlocal fractional boundary value problem. Nonlinear Anal 2010, 72:916-924.

30. Ahmad B, Sivasundaram S: Existence of solutions for impulsive integral boundary value problems of fractional order. Nonlinear Anal Hybrid Syst 2010, 4:134-141.

31. Mophou GM: Existence and uniqueness of mild solutions to impulsive fractional differential equations. Nonlinear Anal 2010, 72:1604-1615.

32. Agarwal RP, O'Regan D, Stanek S: Positive solutions for Dirichlet problems of singular nonlinear fractional differential equations. J Math Anal Appl 2010, 371:57-68.

33. Wang G, Ahmad B, Zhang L: Impulsive anti-periodic boundary value problem for non-linear differential equations of fractional order. Nonlinear Anal 2011, 74:792-804.

34. Zhang L, Wang G: Existence of solutions for nonlinear fractional differential equations with impulses and antiperiodic boundary conditions. EJ Qual Theory Differ Equ 2011, 7:1-11.

35. Ăleanu DB, Trujillo Jj: A new method of finding the fractional Euler-Lagrange and Hamil-ton equations within Caputo fractional derivatives. Commun Nonlinear Sci Numer Simulat 2010, 15:1111-1115.

36. Ăleanu DB, Mustafa OG, Agarwal RP: An existence result for a superlinear fractional differential equation. App/ Math Lett 2010, 23:1129-1132.

37. Agarwal RP, O'Regan D, Wong PJY: Positive Solutions of Differential, Difference and Integral Equations, Kluwer Academic Publishers, Dordrecht 1999.

38. Burton TA: Differential inequalities for integral and delay differential equations. In Comparison Methods and Stability Theory. Lecture Notes in Pure and Applied Mathematics. Dekker, New York Edited by: Liu X, Siegel D 1994.

39. Augustynowicz A, Leszczynski H, Walter W: On some nonlinear ordinary differential equations with advanced arguments. Nonlinear Anal 2003, 53:495-505.

40. Yan JR: Oscillation of first-order impulsive differential equations with advanced argument. Comput Math Appl 2001, 42:1353-1363.

41. Yang Ch, Zhai Ch, Yan J: Positive solutions of the three-point boundary value problem for second order differential equations with an advanced argument. Nonlinear Anal 2006, 65:2013-2023.

42. Jankowski T: Positive solutions for fourth-order differential equations with deviating arguments and integral boundary conditions. Nonlinear Anal 2010, 73:1289-1299.

43. Jankowski T: First-order advanced difference equations. Appl Math Comput 2010, 216:1242-1249. 
44. Wang G, Zhang L, Song G: Integral boundary value problems for first order integro-differential equations with deviating arguments. J Comput Appl Math 2009, 225:602-611.

45. Wang G: Boundary value problems for systems of nonlinear integro-differential equations with deviating arguments. J Comput Appl Math 2010, 234:1356-1363.

46. Balachandran K, Park JY, Julie MD: On local attractivity of solutions of a functional integral equation of fractional order with deviating arguments. Commun Nonlinear Sci Numer Simulat 2010, 15:2809-2817.

47. Wang G, Zhang L, Ntouyas SK: Multiplicity of positive solutions for fractional-order three-point boundary value problems. Commun Appl Nonlinear Anal, (to appear).

48. Ntouyas SK, Wang G, Zhang L: Positive solutions of arbitrary order nonlinear fractional differential equations with advanced arguments. Opusc Math 2011, 31:433-442.

49. Guo D, Lakshmikantham V: Nonlinear Problems in Abstract Cones. Academic Press, New York; 1988

doi:10.1186/1687-1847-2011-2

Cite this article as: Wang et al:: Positive solutions of the three-point boundary value problem for fractional-order differential equations with an advanced argument. Advances in Difference Equations 2011 2011:2.

\section{Submit your manuscript to a SpringerOpen ${ }^{\circ}$} journal and benefit from:

- Convenient online submission

Rigorous peer review

- Immediate publication on acceptance

- Open access: articles freely available online

- High visibility within the field

- Retaining the copyright to your article

Submit your next manuscript at $\boldsymbol{s p r i n g e r o p e n . c o m ~}$ 